8

\title{
Specifying the ODP Trader: An Introduction to E-LOTOS
}

\author{
Giovanny F. Lucero
}

Dept. of Informatics, Federal University of Pernambuco

P.O. Box 7851, CEP 50732-970 Recife - Brazil

gfp@di.ufpe.br

Juan Quemada

Dept. of Telematics Engineering, Technical University of Madrid

ETSI Telecomunicación (B-202), E-28040 Madrid - Spain

quemada@dit.upm.es

\begin{abstract}
An E-LOTOS specification of the computational viewpoint of the ODP trader is presented. E-LOTOS is a new FDT being standardized in the ISO ODP framework. The trader specification is on one hand an assessment of the capabilities of E-LOTOS in specifying ODP elements, but is on the other hand an introduction to the most relevant features of E-LOTOS. E-LOTOS maintains the spirit and the most fundamental operators of LOTOS. It has includes a new functional data typing language, as well as a rich set of new elements such as time, exception handling, a generalized sequential operator, a more general parallel composition operator, gate typing, partial synchronization,...
\end{abstract}

LOTOS, E-LOTOS, ODP trading.

\section{Keywords}

\section{INTRODUCTION}

LOTOS (ISO89 1989) is an ISO standard which has been successfully used to produce formal description of a great variety of applications such as communication protocols, reactive systems, phone systems, ..., as well as to de- 
sign real implementations (Turner 1993, Boumezbeur et al. 1993, Faci et al. 1993, Fernández et al. 1992). Its success is due to two main facts: 1) It is mathematically well-defined, but has nevertheless a very high expressive power; 2)It supports many parts of the software/system design cycle such as abstraction, a variety of design styles, a stepwise refinement approach, a mathematical framework for verification and testing, tools that support most of the parts of the life cycle, ....

A question raised in 1991 asking to enhance the existing LOTOS Standard led to the creation of a Work Item (WI) on "Enhancements to LOTOS" in 1993. The reasons for enhancement were the lack of some technical features, such as user friendly and executable data types, modules, time, gate typing, .... The WI has analyzed during its life time a large number of enhancement proposals. A decision was taken in May 1996. The resulting language has a reasonable increase in complexity (compared to LOTOS). It includes the following enhancements: executability of data expressions, user friendly data types with predefined types, modules, subtyping, partial functions, time, exceptions and exception handling, gate typing and partial synchronization, .... The rich variety of specification styles and the abstraction capabilities of LOTOS is even enlarged with the new enhancements. The WI has proposed the progression of the work to an independent standard which is usually referenced as E-LOTOS (ISO/IEC JTC1/SC21/WG7 1997). The definition has passed the CD Ballot with the support of 11 countries and will be submitted for FCD balloting by the end of the Summer 1997. The basic structure of ELOTOS has been defined, but some adaptation may occur before E-LOTOS reaches its final status.

In this paper, we present an E-LOTOS specification of the ODP trader from the computational viewpoint. The ODP trader is a paradigm of a broker object which links clients and service providers in an open and dynamically changing distributed system. A trader models the procedures to be followed, on one hand by service providers to announce the services offered and on the other hand by users to find service offers. The trader specification is therefore a good example of how E-LOTOS can be applied to specify real ODP systems. The specification given follows the computational description of the trading function given in ISO/IEC DIS 13235 (1995) which is a non formal textual description. The specification describes the most relevant parts of the functionality given in the informal description. Most features not considered in the formal specification can be added directly without difficulty.

In the remainder of the paper we assume that the reader is familiar with LOTOS. We give explanations only of the new features which do not exist in LOTOS. The paper can be used therefore as an introduction for LOTOS users of the most relevant new features of E-LOTOS.

The paper is structured as follows: Section 2 summarizes the changes and enhancements introduced in E-LOTOS with respect to the original version of LOTOS. Section 3 provides an informal introduction of the trading function. 
Section 4 includes the most relevant parts of the E-LOTOS specification of the ODP trader complemented with more generic descriptions of the most important parts of E-LOTOS. This explanations should provide newcomers a deeper insight into the capabilities of the new language. Section 5 describes the conclusions obtained by the authors about this specification experiment and about E-LOTOS as a specification language. Finally, section 6 acknowledges the rest of the regular members of the E-LOTOS WI which have participated in the design of E-LOTOS.

\section{E-LOTOS}

E-LOTOS (ISO/IEC JTC1/SC21/WG7 1997) is formed of a behavioral process algebra part, which generalizes the basic LOTOS algebra in several directions but which keeps most LOTOS operators as particular cases of the new ones, and an executable and user friendly functional data definition language.

A list is given now which tries to highlight from the user point of view the most notable new features from which an E-LOTOS user should benefit when using the language.

- A loop construct which avoids the need for implemented cyclic behaviours with recursive processes has been introduced. This is the only place where multiple assignment of a variable is allowed in each iteration of the loop.

- Output variables in processes and functions (Garavel et al. 1996). The use of output variables in processes and functions together with the new sequential composition operator provides a much more readable and concise way for value communication in sequential compositions. The following example illustrates the way assignment, functions and processes communicate through input and output values

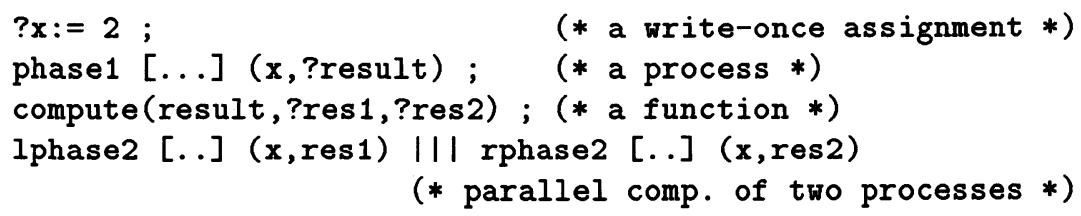

- A general parallel n-ary operator which supports synchronization of $\mathbf{J}$ processes among $K$ processes $(J \leq K)$ (Garavel et al. 1996). Synchronization patterns of 2 processes among $\mathrm{N}$ are possible. The new parallel operator is clear and readable and has the LOTOS operators as particular cases.

- A Suspend/Resume operator which generalizes disabling by allowing a disabled behaviour to be resumed by a specific action of the disabling behaviour (Logrippo et al. 1994, Hernalsteen 1996).

- Exceptions and exception handling in the behavioural and data parts with a 
uniform approach. Exception mechanisms permit new ways of structuring and are demanded by ODP (Quemada et al. 1992, Garavel et al. 1996, Jeffrey 1996).

- Explicit renaming operator for observable actions or exceptions (Jeffrey et al. 1996). The renaming operator allows not only to change the name of the events occurring but to add and remove fields from the structure of events, or to merge and to split gates.

- Gates must be explicitly typed with a given record structure. Partial synchronization can be achieved when the gate types satisfy a subtyping relation in addition to value matching on the common part. Partial synchronization allows very elegant constraint oriented specifications (Jeffrey 1996, Garavel 1994, Mañas 1994, Najm et al. 1995).

\section{AN OVERVIEW OF THE ODP TRADER}

In order to use services in a open distributed systems, users need to know which services are available and who are their providers. Since sites and applications are frequently changing in large distributed systems, it seems compulsory to have a mechanism which enables software components to find appropriate services providers. This mechanism, called Trading Function, is supplied in ODP (ISO/IEC DIS 10746 1995) by the trader object.

Following the philosophy of ODP, the trader is specified through several viewpoints. In the next sections, we gives an informal description of these viewpoints.

\subsection{Enterprise viewpoint}

From the enterprise viewpoint, a trader is an object that enables clients to find dynamically suitable servers in an ODP system. A trader can be viewed as an advertiser where objects can announce their capabilities and become aware of capabilities of other objects.

An announcement in a trader is called service offer. It describes the characteristics or properties satisfied by the service. In addition to service properties, a service offer also contains the interface where the service is available.

Advertising a service offer is called export. When a trader accepts an export request, it stores the exported service offer in a centralized or distributed database. This database is often termed service offer space.

On the other hand, an importer can require knowledge about adequate service providers. In this case, the trader accepts a request, called import, containing an expression of service requirements desired by the importer. The trader matches the importer's service request with its database of service offers and selects a list (possibly empty) of appropriated service offers which satisfy the requirements made by the importer. 


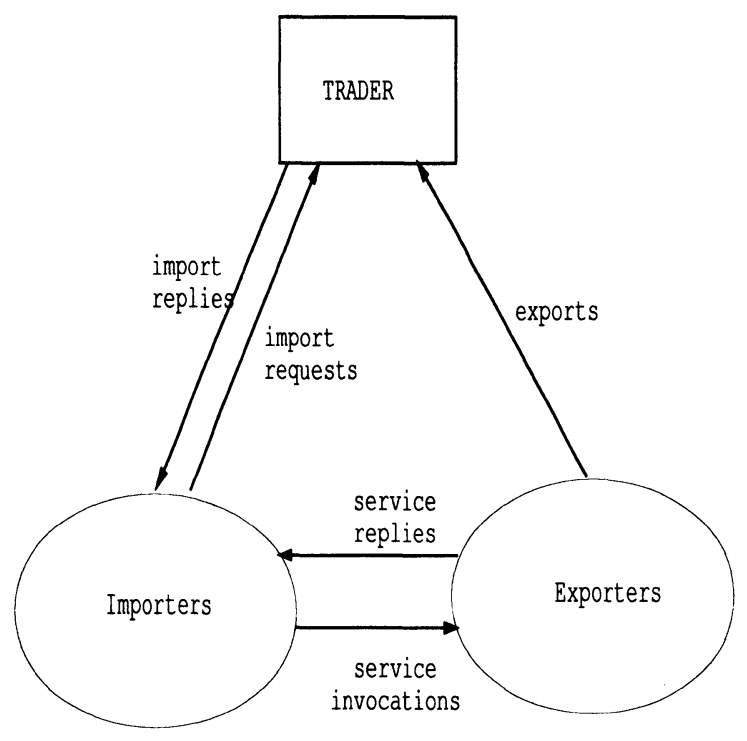

Figure 1 Interactions of a trader and its clients

The list of matched services offers is returned to the importer which may then interact directly with any service described in the list. Figure 1 summarizes the interactions of a trader and its clients.

Export and import activities are governed by a trading policy, which comprises trader policies, importer policies and exporter policies. Where an activity involves interactions between objects, the resulting policy will be a compromise between the wishes of the interacting objects. Therefore, a trader's behaviour is limited by the policies established for these activities. In other words, trader policies determine and guide a trader's behaviour. For example, a trader policy can restrict resources used by an individual import request.

Several autonomous traders can be "linked" in order to share their service offer spaces. Thus, a trader also can play the role of exporter or importer with respect to other trader(s). Such a group of autonomous traders is termed interworking group. A trader within an interworking group enlarges the service offer space for its users by including offers of other traders in the group. This enlargement of the service offer space is made indirectly when a trader propagates import requests to neighbor traders.

\subsection{Information viewpoint}

The trader information viewpoint defines the information elements and the relationships between them which are manipulated by the ODP trading func- 
tion. In the standard ISO/IEC DIS 13235 (1995), this viewpoint is formally described using the formal specification notation $\mathrm{Z}$. The specification includes basic concepts for information and static invariant and dynamic schemata for the ODP trading function. In this paper we do not consider this viewpoint.

\subsection{Computational viewpoint}

The trading function computational viewpoint describes an object template for a trader. This object has interfaces for service and management operations. Service operations are related to import and export activities whereas management operations are provided to add, delete or modify links to other traders.

In the standard ISO/IEC DIS 13235 (1995), this viewpoint description comprehends:

- signature templates for the service interface and management interface (defined in CORBA IDL),

- types used in the operations parameters (defined in CORBA IDL), and

- informal descriptions of the trader's behaviour.

The trader's behaviour is given by the behaviour of every service operation and management operation, plus a set of constraints on interleaving of actions performed by these operations. In the next section, we give in E-LOTOS a formal description of the trader's behaviour. This formal specification could be viewed as a complement of the informal one given in ISO/IEC DIS 13235 (1995).

\section{E-LOTOS SPECIFICATION OF THE TRADER}

This section outlines our E-LOTOS specification of the ODP trader's computational viewpoint. We present only the most relevant parts of the specification. In particular, we pay attention to the definition of the trader's behaviour. The reader interested in the complete specification is referred to the annex $\mathrm{A}$ of ISO/IEC JTC1/SC21/WG7 (1997).

\subsection{The structure of a trader community}

A trader is modeled by a process which can interact with its environment through two ports. On one port, the trader receives operation invocations from clients and management objects. On the other port, it returns the re- 
sults to the respective invoker. Following the ODP terminology, we will call termination the action of returning a result.

In a trading community, every object (trader or client) has an interface identifier. An object in this community uses these identifiers in order to refer to the other objects. Thus, invocations and terminations are represented by events having respectively the following schemes

inv (! id !origId !...operation parameters...)

ter (!id !origId !...termination results...)

where id identifies the invoked trader and origId identifies the object which requests the operation. The trader receives invocations on the gate inv and sends the respective terminations on the gate ter.

A trading community can be described using the new E-LOTOS operator par. This operator allows to specify structurally and clearly architectures of systems which are difficult or, sometimes, impossible to express in LOTOS. Thus for example, the following E-LOTOS code shows a scenario where a trader communicates directly with an importer object and indirectly, through a binding object, with an importer-exporter object. The binding object redirects invocations and terminations between objects.

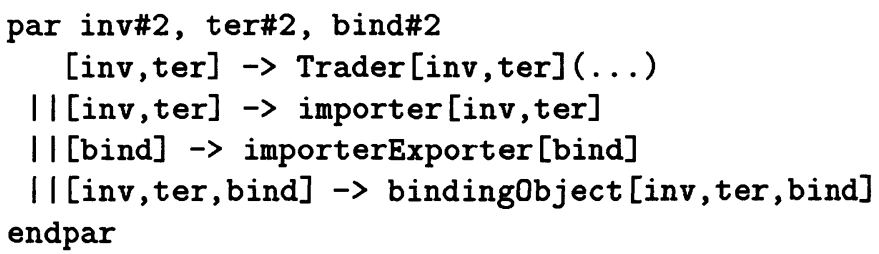

The code above specifies that communication in the gates tagged with \#2 is one-to-one, i.e., processes synchronize by pairs on the gates listed in the square brackets before the respective “->". Notice that the style of specification used in this piece of E-LOTOS code is resource oriented. The par operator provides better flexibility and expressiveness than the three original parallel operators joined $(|[\ldots]|,||$ and || $\mid)$. Specification styles like constraint oriented and resource oriented, which use parallel composition extensively, greatly benefit by this E-LOTOS extension.

\subsection{Type declarations}

In the specification, the greatest part of the type declarations comprises types for the operation parameters. The standard (ISO/IEC DIS 13235 1995) defines these types in CORBA IDL and their translation to E-LOTOS is very easy. Also, we translate exception declarations given in CORBA IDL to type 
declarations in the E-LOTOS notation. In order to make more concise the specification, we have removed the tailing string "Type" from all type names in ISO/IEC DIS 13235 (1995).

Ports are typed in E-LOTOS, therefore we need to specify types for data exchanged (offered) by E-LOTOS processes. In particular, we need to specify which are the types involved in trader interactions.

Data types in E-LOTOS are declared as in SML but using records rather than tuples in the constructor arguments. Thus, the signature for operation invocations is defined by the following type:

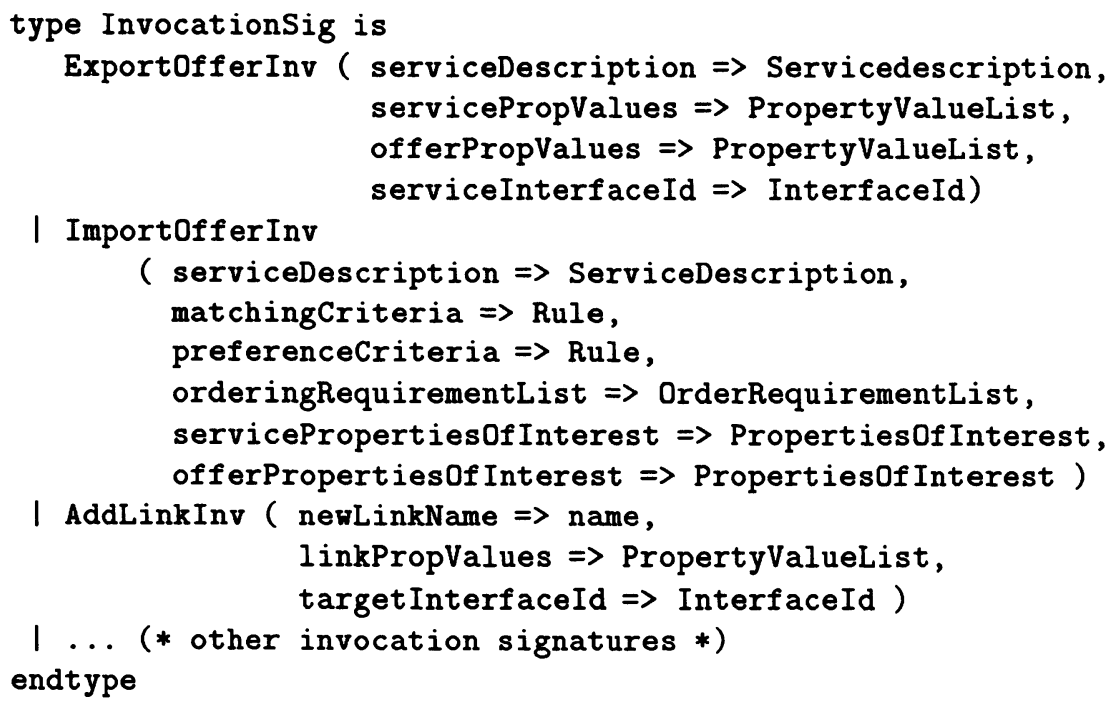

This declaration introduces a union type and its constructors. Each branch separated by "I" defines one constructor and the type of its arguments. The type of the arguments is given by a record type using the "=>" notation to define named fields. The name of the field and its type are separated by the "=>" symbol.

An invocation signature states the input parameters of the respective operation. Thus, the signature for export operation invocations has a description of the service which will be exported, a list with the properties of the service, a list with the properties of the service offer, and a identifier of the interface where the service will be provided. A property of a service might be the cost of the service. A property of a service offer might be the date when the offer expires.

An import operation invocation contains a service description of the desired service, a rule to constrain the matching of suitable servers, a rule to indicate preferred services and some indications for the formatting of results. Rules are used to define politics of search in an import operation. A rule is expressed 
by a predicate over the properties of the desired service and offer service. For example, a rule expressing matching criteria might be "the cost of the service is less than $\$ 10 "$ ". Rules are modeled using recursive data types. By lack of space we do not include their definition here.

The operation AddLink, which allows to create links between traders, has a invocation signature with the name of the link, a list of properties of the link and a identifier of the target trader.

For each kind of invocation, a proper kind of termination is needed. We define termination signatures for trader operations by

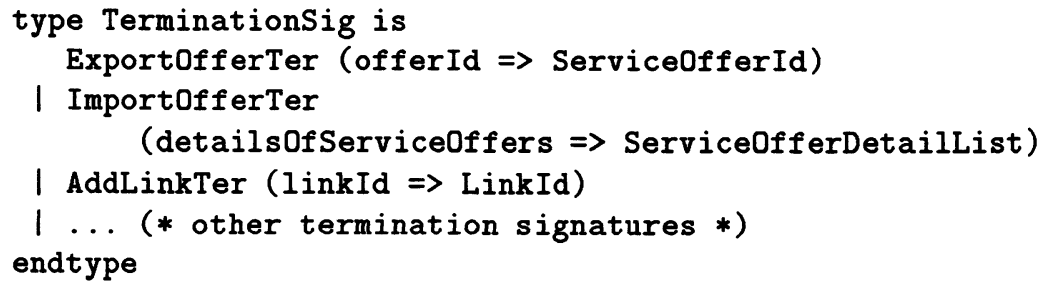

A termination signature states the output parameters for a given operation. The export offer operation returns a new identifier for the recently exported service offer. The import operation returns a list detailing the properties of suitable (matched) services. An add link operation returns a new identifier for the link added by the performance of this operation.

The data exchanged for trading are invocations and terminations. The type of this data is defined below using record types. We define the type Invocation (termination) for typing gates that exchange invocations (terminations) of trader operations. The type Invocation (Termination) is a record with three fields: the invoked trader identifier, the originator client identifier and the input (output) parameters of the operation.

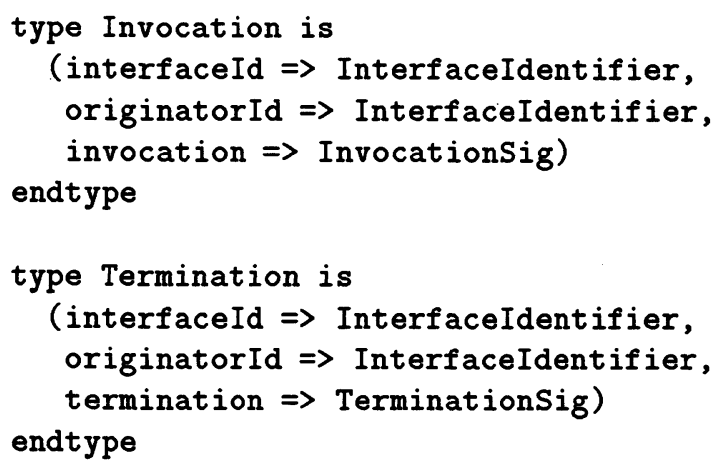




\subsection{Function declarations}

In this section, we only show some simple examples of function declarations. The examples illustrate the functional language used in E-LOTOS to write data expressions.

The following declaration defines a function which allows to see if a (property) name occurs in lists of name-value pairs.

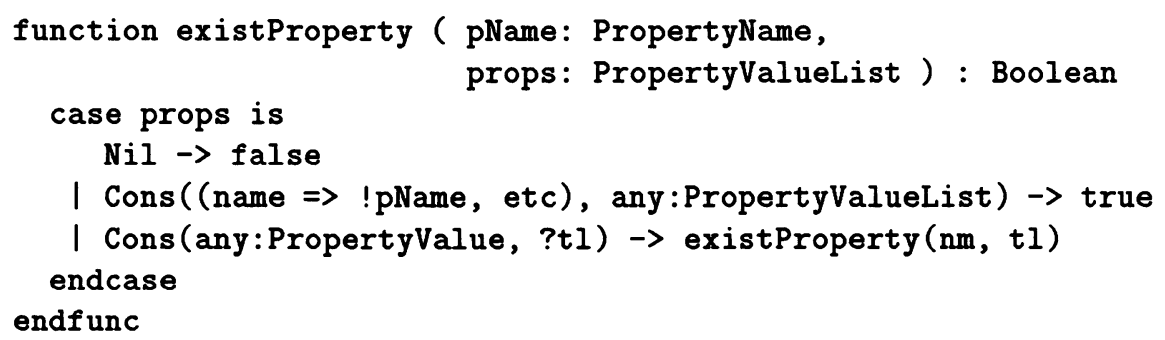

Notice some details in the above the definition. The case constructor uses pattern matching. In patterns, binding occurrences of variables are decorated with "?" and expressions decorated with "!" denote values offered. This syntax is in accord with the syntax of events. The occurrence of "etc" is not meta-linguistic. The "etc" notation completes the record pattern and denotes the remaining components of the record.

The following specification takes advantage of the exception mechanism introduced by E-LOTOS in order to define some needed partial functions. For example, the following propValue0f function receives a property name and a list of property name-value pairs. When the name appears in the list, it returns the value associated to the name in the list. In the other case, the function raises an exception.

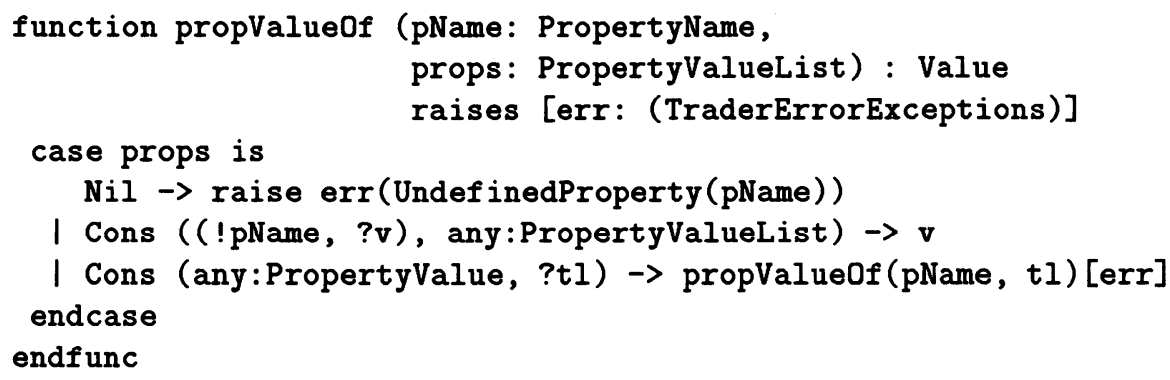




\subsection{Computational behaviour of the trader}

Like LOTOS, E-LOTOS makes it possible to describe the behaviour of systems in a stepwise fashion, moving from one abstraction level to another. Each level can be specified in different specification styles. We follow this methodology in our specification. At the most abstract level, the trader object is a parallel composition of three resources (processes). The ServiceInterface and ManagementInterface processes provide functionality for service operations and management operations, respectively. The process StateProc represents the trader state.

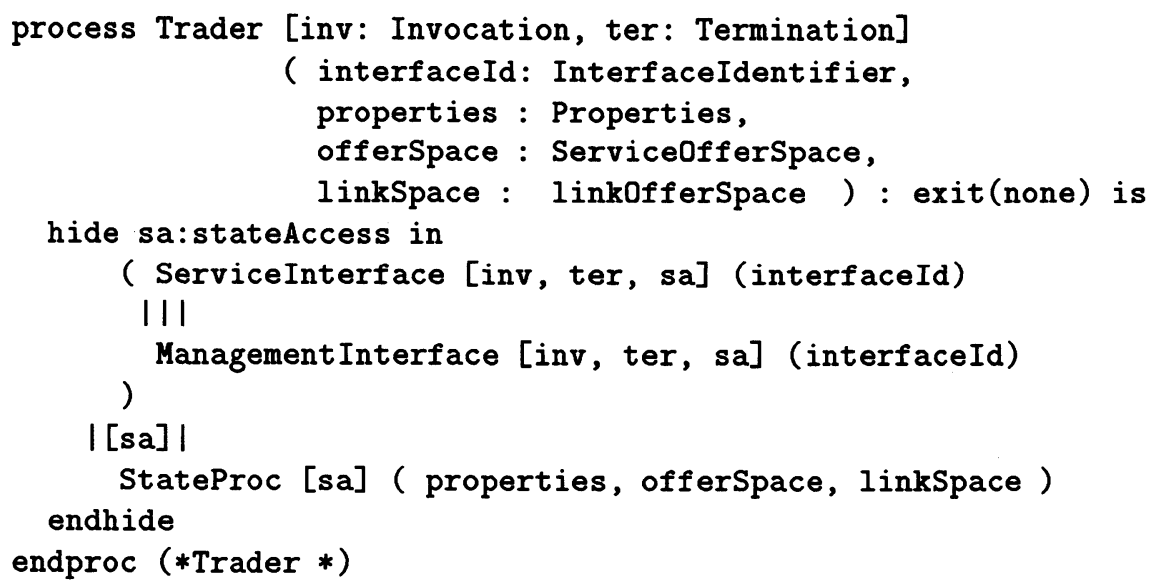

Service operations are related to export and import activities and they only write in the trader service offer space. Management operations manipulate connections between interworking traders updating trader links. Therefore, service operations and management operations can be performed in parallel without destroying the consistency of the trader state.

The process StateProc encapsulates three elements which conform the trader state: a set of trader properties, the service offer space and the link space. Service and management operations use the trader state through the port sa which is hidden within the trader.

In order to illustrate how the operation's behaviour is defined we show the definition of the ServiceInterface process. In this process, all service operations are offered to clients in parallel. However, the availability of operations is constrained in such way that accessing operations (as import offer) and modifying operations (as export offer) can not be overlapped in time. Availability of operations is defined in a constraint oriented style by composing the processes representing operations with the OrderingConstraints process. 


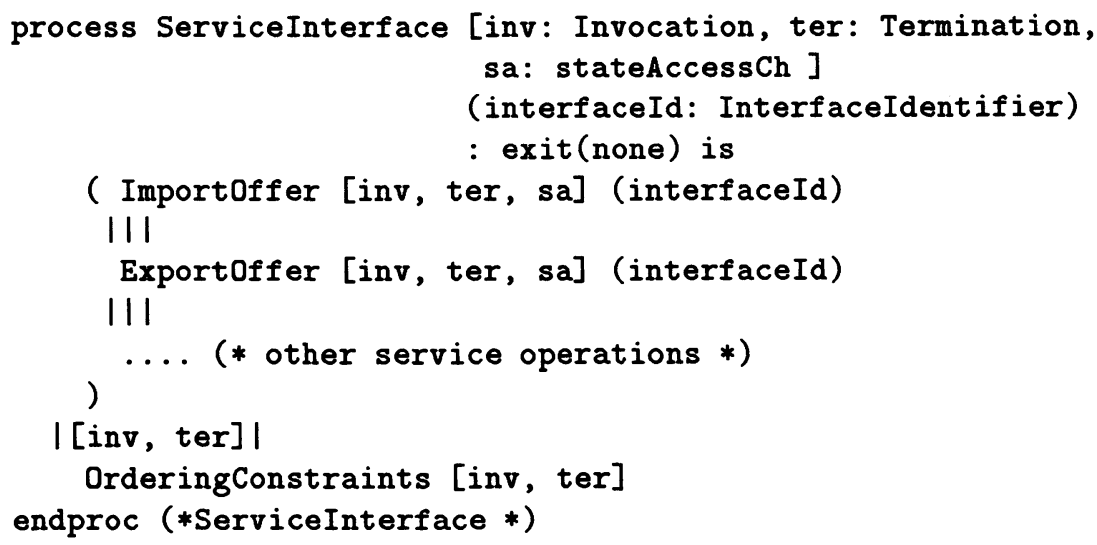

In one more step of refinement we define below the Exportoffer and Import0ffer processes. The Exportoffer process enables clients to perform offer exports. For each invocation, the process interacts with the state to update the offer database adding the new offer and then, in the termination, it replies with the fresh identifier assigned to the offer. Afterwards, when the offer expires, the management object is notified about the expiration.

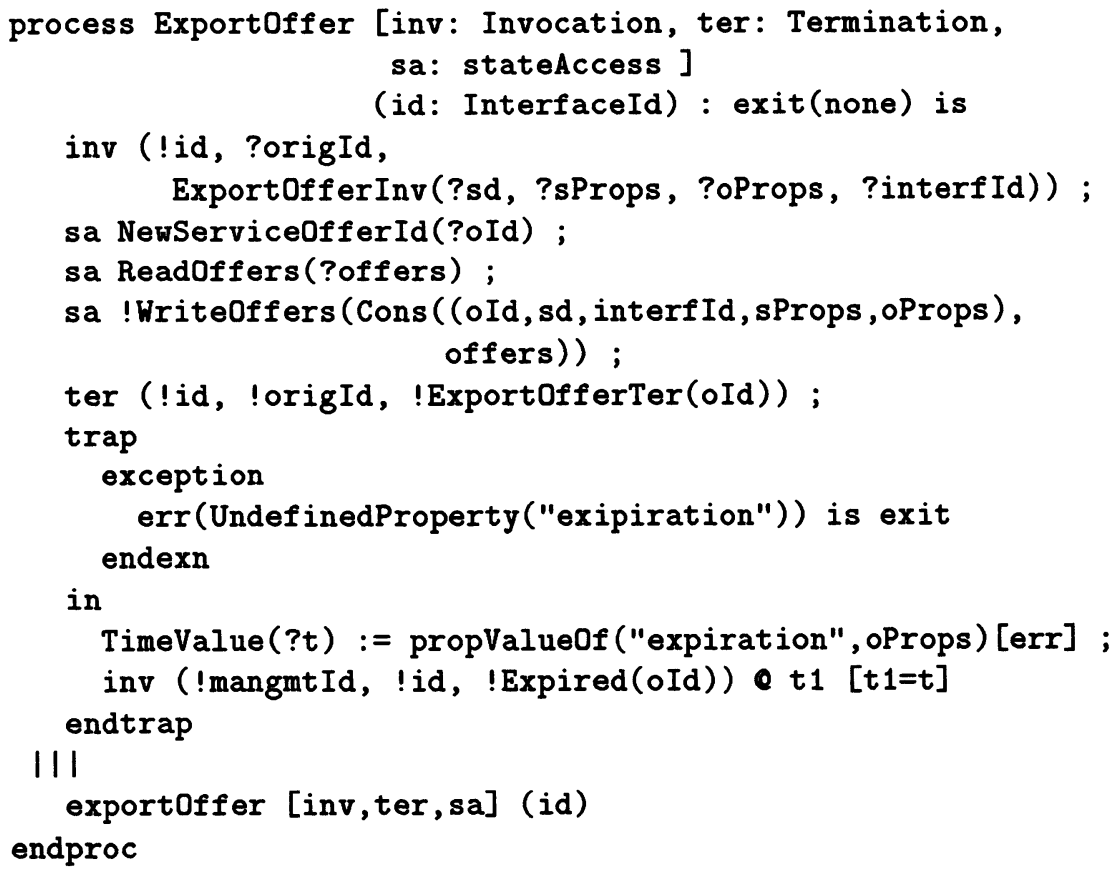


This process defines a behaviour which replicates infinitely the behaviour of one export. This infinite process represents the possibility of simultaneously accepting of several exports. However, the availability of exports is constrained by the OrderingConstraints in the service interface (see the process ServiceInterface above).

The behaviour representing one export sequentially perform the following actions: accepts an export offer invocation, gets a fresh offer identifier, writes the offer in the offer space, replies to the exporter and finally, if the offer has an expiration property, notifies the management object at time of expiration.

In this last definition above we use some new concepts introduced by ELOTOS: assignment, exception trapping and explicit time restrictions. The left part of the assignment ":=" is a pattern which is matched with the evaluation of the right part.

The trap constructor allows to capture and to handle exceptions raised when expressions are evaluated. Thus for example, the evaluation of the function propValue0f will raise an exception when the expiration property is undefined. In this case, the exception is trapped and handled by an exit.

The 0 t 1 [t $1=t]$ construction in the last inv ... action occurrence restrict the performance of the action at a given time. Thus, when the invocation is performed, $t 1$ is bound to the current time hence the guard [ $t 1=t]$ constraint its realization at time of the offer expiration.

The definition of the process Exportoffer exemplifies the use of the real time capabilities of E-LOTOS which are strongly based on the proposal for real-time LOTOS described in Léonard et al. (1997). Here, we use time to define timeouts to signal offer expirations. Real time is important also in other applications. In ODP, for example, it can be used to describe features like QoS of binding objects.

Notice that the actual deletion of expired offers will depend on the management object policy therefore it is not specified here. For example, it can dictate that expired offers must be deleted immediately. Another policy could be to leave expired offers in the offer database until new exports demand the space occupied by them.

In addition, it is worth to note the readability of the definition. It is reached through the use of E-LOTOS proper constructs like assignment and traps. The reader who would attempt to write a similar specification in LOTOS would perceive a considerable loss of readability.

Another significant service operation is the import of an offer. The behaviour of the operation is given below by the Importoffer process definition.

Informally, for each invocation, the Importoffer process performs the following activities: receive the invocation, access the state to get the offer database, trader links and trader properties, match the offers against arguments and propagate the import invocation to interoperate with "neighbor" traders and, when some trader finds the right matches, they are submitted in the termination. 


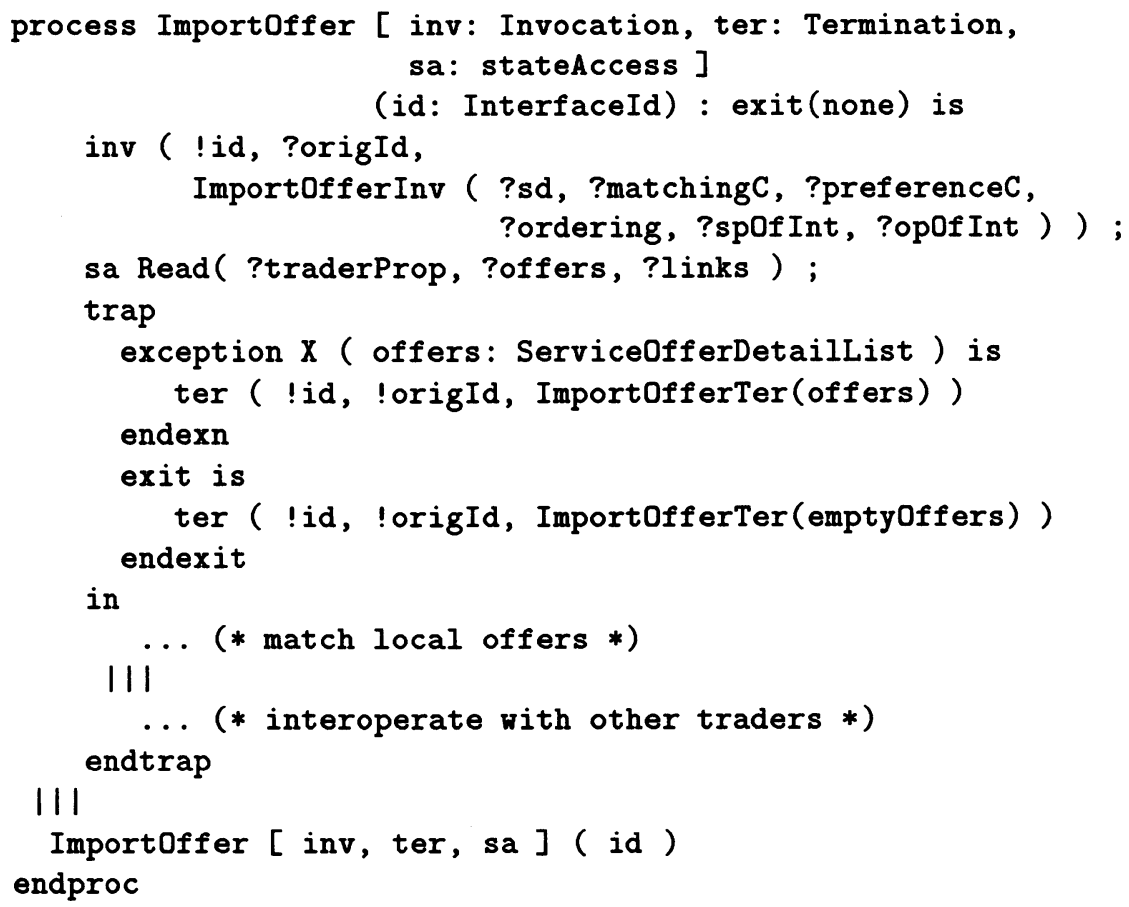

The above definition is a good example to illustrate a non-orthodox use of the trap constructor. In general, the trap is used as a tool for exception handling. However, it also increase the expression power allowing to define new behaviour schemes. Here, for example, we use this constructor to express that, when several traders are interoperating in an import operation, the matched offers returned to the original importer are those of the "first" trader which was successful in the matching activity.

\section{CONCLUSIONS AND FURTHER WORK}

This paper provides a brief introduction to E-LOTOS and to its usage in specifying the ODP Trader. This is one of the first applications of this new FDT to a medium size example. E-LOTOS and the new enhancements have proved to be suitable for the description of the trader. The use of time, new parallel, exceptions and new typing facilities makes the specification shorter, more readable, more concise and allows a better structuring. The usage of the enhancements introduced has only started to be understood, but the results are very encouraging.

The definition of E-LOTOS is now in its final stages and examples as this one provide the basis for the last tuning. The standard is now in the last stages of the process where the last (small) changes can and probably will be 
introduced. Of course the core of E-LOTOS should be stable in the future. Changes in the syntax or in the static semantics may still occur. The main motivation for such changes will be, the alignment with ODP standards such as CORBA or IDL, the production of a uniform syntax which can be properly parsed, the removal of inconsistencies or errors in the present definition,...

\section{ACKNOWLEDGEMENTS}

The development of E-LOTOS has been a collective effort in which many contributors have participated. The work in ISO is performed within Work Items (WI) where national experts contribute and progress the work. One of the co-authors, Juan Quemada, has coordinated and chaired the "Enhancement to LOTOS" WI rapporteur and editor and he would like to acknowledge the work performed by many other national experts which have also contributed with his work, ideas and contributions to the definition of E-LOTOS. Among all the participants in the WI, the ones which have had a regular participation with contributions during some phase of the work are: Arnaud Février, Hubert Garavel, Alan Jeffrey, Guy Leduc, Luc Leonard, Luigi Logrippo, Jose Manas, Elie Najm, Mihaela Sighireanu and Jacques Sincennes.

This work has been jointly supported by Spanish National Research Program - the CICYT project DISC and CNPq/Brazil.

\section{REFERENCES}

ISO 8807 (1989) LOTOS-A formal description technique based on the temporal ordering of observational behaviour, International Standard.

ed. K.J. Turner (1993), Using Formal Description Techniques, an introdution to Estelle, LOTOS and SDL, Jonh Wiley \& Sons.

Boumezbeur, R. and Logrippo, L. (1993) Specifying telephone systems in LOTOS, in IEEE Communications Magazine, August.

Faci, M. and Logrippo, L. (1993) Specifying hardware systems in LOTOS, in Proc. of Computer Hardware Description Languages and their Applications XI (CHDL'93), North-Holland.

Fernández, A. and Miguel, C. and Vidaller, L. and Quemada, J. (1992) Development of Satellite Communication Networks based on LOTOS, in Protocol Specification, Testing and Verification XII, North Holland, Amsterdam.

ISO/IEC JTC1/SC21/WG7 1.21.20.2.3 (1997) Working Draft on Enhancements to LOTOS, ISO Working Group 7, Also available by ftp at ftp://ftp.dit.upm.es/pub/lotos/elotos/Working.Docs/cd.ps.gz.

ISO/IEC DIS 13235 (1995) ODP Trading Function.

Garavel, H. (1994) On the Introduction of Gate Typing in E-LOTOS, VERIMAG, France. 
Garavel, H. and Sighireanu, M. (1996) A Wish List for the Behaviour Part of LOTOS, VERIMAG, France.

Logrippo, L. and Steppien, B. (1994) Specifying the Suspend/Resume Operator, University of Ottawa.

Hernalsteen, C. (1996) Introduction of a Suspend/Resume Operator in ELOTOS, ENST, France.

Quemada, J. and Azcorra, A. (1992) Structuring Protocols with Exception in a LOTOS Extension, in PSTV XII, Orlando.

Jeffrey, A. (1996) A Core Data and Behaviour Language for E-LOTOS, University of Sussex.

Jeffrey, A. and Leduc, G. (1996) E-LOTOS Core Language, University of Sussex.

Mañas, J. (1994) Typed Gates, DIT-UPM, Spain.

Najm, E. and Février, A. (1995) Extending Gate Typing to Mobile LOTOS, ENST, France.

ISO/IEC 10746 (1995) Open Distributed Processing - Part 1-4.

Léonard, L. and Leduc, L. (1997) An introduction to ET-LOTOS for the description of time-sensitive systems, Computer Networks and ISDN Systems, 29 3, 271-292.

\section{BIOGRAPHY}

Giovanny Lucero is currently a Guest Professor at Department of Computer Science of the Federal University of Sergipe and a $\mathrm{Ph}$. D. student at the Federal University of Pernambuco (UFPE) at Brazil. He obtained a M.Sc. in Computer Science at UFPE in 1993 and his main research interest is the semantics of programming languages, and especially the overlap between functional programming and theories of objects and concurrency.

Juan Quemada is currently Professor at the Telematics Engineering Department of the Universidad Politecnica de Madrid, from where he achieved his Telecommunication Engineering Degree and a Phd in Telecommunication Engineering in 1981. He gives courses and conferences regularly and has authored over a hundred technical publications, including contributions in over 30 co-authored books. He is a regular member of the program committee of scientific conferences such as FORTE, PSTV, FMOODS or ICODP. With about twenty years of experience in the area of telecommunications and distributed systems his present focus of activity is in the use of CSCW in new service creation and in the use of formal methods in the design of communication and distributed systems. As rapporteur and editor of the WI on "Enhancements to LOTOS" he has been coordinating the definition of the E-LOTOS Standard in ISO/IEC. 